\title{
ALCAM regulates multiple myeloma chemoresistant side population
}

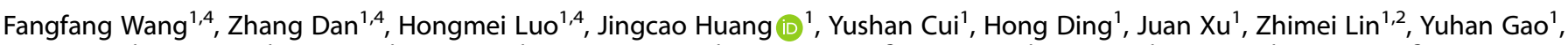
Xinyu Zhai ${ }^{1}$, Yan Yang ${ }^{1}$, Ying $\mathrm{Qu}^{1}{ }^{1}$, Li Zhang ${ }^{1}$, Fengjiao Chen ${ }^{1}$, Qiang Wang ${ }^{3}$, Xin Wang ${ }^{1}$, Yu Feng ${ }^{1}$, Ting Liu ${ }^{1}$, Qing Yi ${ }^{3}$, Ting Niu ${ }^{1 \times}$ and Yuhuan Zheng (iD ${ }^{1 凶}$

(c) The Author(s) 2022

Drug-resistance is a major problem preventing a cure in patients with multiple myeloma (MM). Previously, we demonstrated that activated-leukocyte-cell-adhesion-molecule (ALCAM) is a prognostic factor in MM and inhibits EGF/EGFR-initiated MM clonogenicity. In this study, we further showed that the ALCAM-EGF/EGFR axis regulated the MM side population (SP)-mediated drug-resistance. ALCAM-knockdown MM cells displayed an enhanced ratio of SP cells in the presence of bone marrow stromal cells (BMSCs) or with the supplement of recombinant EGF. SP MM cells were resistant to chemotherapeutics melphalan or bortezomib. Drug treatment stimulated SP-genesis. Mechanistically, EGFR, primed with EGF, activated the hedgehog pathway and promoted the SP ratio; meanwhile, ALCAM inhibited EGFR downstream pro-MM cell signaling. Further, SP MM cells exhibited an increased number of mitochondria compared to the main population. Interference of the mitochondria function strongly inhibited SP-genesis. Animal studies showed that combination therapy with both an anti-MM agent and EGFR inhibitor gefitinib achieved prolonged MM-bearing mice survival. Hence, our work identifies ALCAM as a novel negative regulator of MM drug-resistance, and EGFR inhibitors may be used to improve MM therapeutic efficacy.

Cell Death and Disease (2022)13:136; https://doi.org/10.1038/s41419-022-04556-8

\section{INTRODUCTION}

Multiple myeloma (MM) is the second most common hematologic malignancy in the US [1]. In the new drug era, with the landmark clinical application of proteasome inhibitors (PIs) and immunomodulatory drugs (IMiDs) in MM treatment, MM is treatable but remains an incurable disease [2]. Drug-resistance widely exists, particularly in some newly diagnosed high-risk MM patients or relapsed/refractory MM patients [3]. However, the mechanism of MM drug-resistance is still not fully understood.

More than a decade ago, Matsui et al. reported heterogeneous cell types in an MM cell line with distinguishable clonogenicity activities [4]. A small population of MM cells, usually $<2 \%$ of the total tumor cells, with clonogenicity capacity was referred to as myeloma cancer stem cells (MM-CSCs) in the same study. Later, the self-renewal, differentiation, and drug-resistant features of MM-CSCs have been demonstrated in many basic studies [5]. MM-CSCs may be identified by different methods, and no consensus of MM-CSC markers or detection methodology currently exists [6]. Hoechst staining is often used to separate MM cells into a side population (SP) and a main population (MP), and the SP MM cells are believed to contribute to MM clonogenesis; therefore, SP MM cells are considered to have "stemness." [7] The drug-resistance feature associated with SP
MM cells has also been investigated [8,9]. More importantly, a recent study using clinical samples suggested a relationship between the stemness features of SP MM cells and MM minimal residual disease [10]. In this work, we investigated the function of activated-leukocyte-cell-adhesion-molecule (ALCAM, also known as CD166) in SP MM cell regulation.

ALCAM is a member of immunoglobulin superfamily proteins [11]. Recently, we demonstrated the ALCAM function in myelomagenesis [12]. ALCAM negatively regulates myeloma clonogenicity. MM patients with high ALCAM expression have superior overall survival. Mechanistically, ALCAM interacts with EGFR and inhibits EGFR downstream pro-MM cell signaling. In this study, we showed that ALCAM regulated MM drug-resistant SP MM cells. Targeting the ALCAM-EGFR/EGF axis might therefore overcome SP MM-mediated drug-resistance.

\section{MATERIALS AND METHODS \\ Primary myeloma samples}

Bone marrow (BM) aspirations from newly diagnosed MM patients were provided by the tissue bank of the Department of Hematology, West China Hospital, Sichuan University, and processed as previously described [12]. This study was approved by the Ethical Committee of West China Hospital of Sichuan University.

\footnotetext{
${ }^{1}$ Department of Hematology, West China Hospital/State Key Laboratory of Biotherapy and Cancer Center, Sichuan University, Chengdu, China. ${ }^{2}$ Department of Hematology, The Affiliated Hospital of Chengdu University, Chengdu, China. ${ }^{3}$ Center for Translational Research in Hematological Malignancies, Cancer Center, Houston Methodist Hospital, Houston, TX, USA. ${ }^{4}$ These authors contributed equally: Fangfang Wang, Dan Zhang, Hongmei Luo. ${ }^{凶}$ email: zhengyuhuan@scu.edu.cn; tingniu@sina.com Edited by Giovanni Blandino
}

Received: 19 October 2021 Revised: 23 December 2021 Accepted: 17 January 2022

Published online: 10 February 2022 


\section{Cell culture}

Human MM cell lines RPMI8226 and MM.1S were maintained in RPMI-1640 medium with $10 \%$ fetal bovine serum (Gemini BioProducts, US), 100 units/ $\mathrm{mL}$ penicillin, and $100 \mathrm{~g} / \mathrm{mL}$ streptomycin at $37^{\circ} \mathrm{C}$ and $5 \% \mathrm{CO}_{2}$. Murine $\mathrm{MM}$ cell line 5TGM1 with consistent luciferase gene expression was maintained in the same culture condition. The cell lines were verified by short tandem repeat analysis and tested for mycoplasma contamination. To generate MM cells with a consistently low ALCAM expression, human MM cell lines RPMI8226 and MM.1S were infected with two different ALCAM shRNA lentiviruses (\#TLHVU2300, Transomic Tech., US).

The following oligonucleotides were used as shRNA sequences to target ALCAM (sh1 5'-CAGAGGAATCTCCTTATATA-3' and sh2 5'-CCGAAGGAATAA GAAGCTCAA- $3^{\prime}$ ). Infected cells were selected and maintained in the culture medium with the addition of puromycin (Sigma Aldrich, US). The control viruses were ordered from Transomic Tech (\#TLHVU2300, Transomic Tech., US). Human bone marrow stromal cells (BMSCs) were derived and maintained as previously described [12].

\section{Antibodies and reagent}

Anti-ALCAM/CD166 (\#343905) antibody for flow cytometry analysis was ordered from Biolegend Inc. Recombinant proteins, including human ALCAM-Fc (\#CD6-H5259) and EGF (\#10605-HNAE), were ordered from Acrobiosystems and Sinobiology Inc., respectively. Anti-EGF (\#AF236) and anti-CD6 (\#AF627) neutralizing antibodies were ordered from R\&D Systems Inc. EGFR inhibitor gefitinib (\#S1025), SMO inhibitor cyclopamine
A
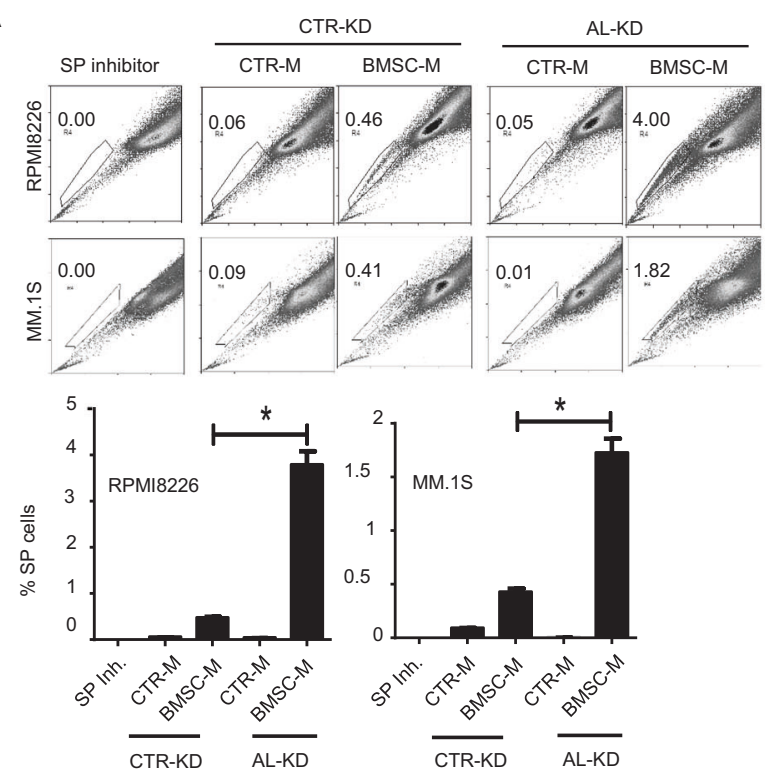

D

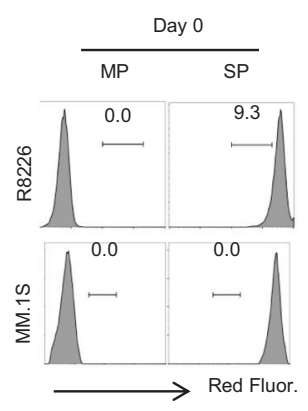

$$
\frac{\text { Day } 4 \text { (with BMSC) }}{\text { CTR-KD AL-KD }}
$$

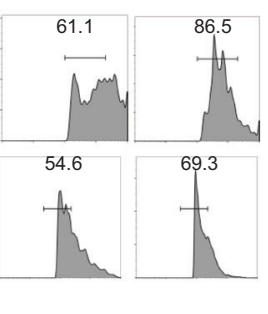

F

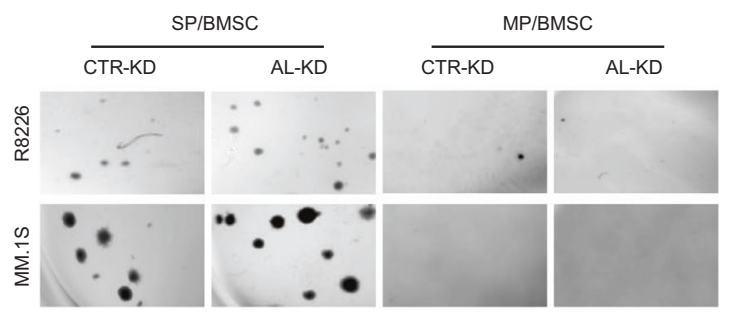

B
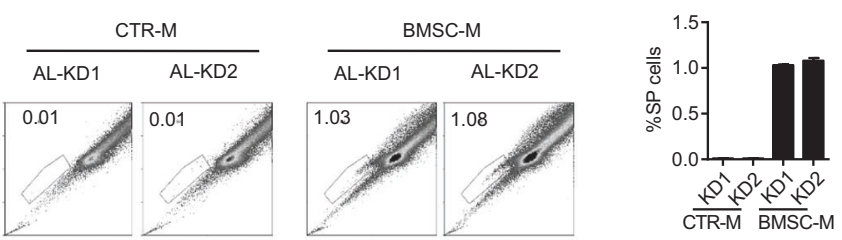

C
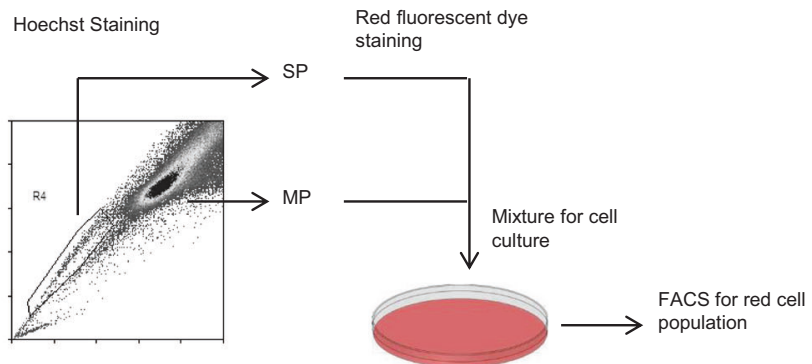

E
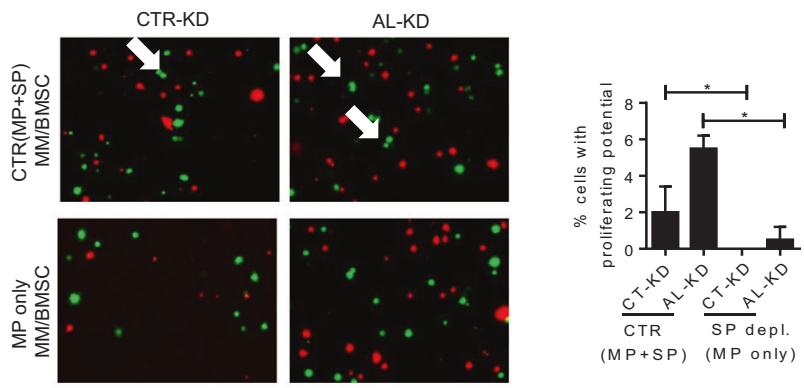

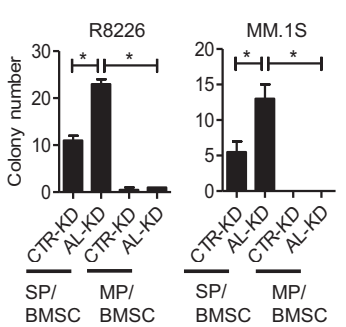

Fig. 1 Bone marrow stroma-derived soluble factors promote ALCAM-knockdown myeloma cell side population. A Hoechst staining of CTR-KD or AL-KD MM cells (RPMI8226 or MM.1S) cultured in regular medium (CTR-M) or BMSC-conditioned medium (BMSC-M) for $48 \mathrm{~h}$. The numbers indicate the percentage of SP cells. B Hoechst staining of AL-KD1 and AL-KD2 RPMI8226 cells cultured in regular medium (CTR-M) or BMSC-conditioned medium (BMSC-M) for $48 \mathrm{~h}$. C Schematic graph: After cell sorting, SP cells were labeled with red fluorescent dye, and MP cells were labeled with CFSE. The labeled SP and MP cells were re-mixed with the ratio (SP:MP = 2:98). D SP cell proliferation was examined by flow cytometry for red fluorescent intensity dilution. E Fluorescence confocal microscopy of MM cells (green) and BMSCs (red) under 3D printing. F Hoechst staining of RPMI8226 cells, followed by cell sorting to isolate SP- and MP-only MM cells. The cells were used for the set of colony formation assay (left panel). Statistical results from the colony formation assays (right panel). Data are the mean of three independent experiments in three replicates. ${ }^{*} p<0.05$. 
(\#S1146), and Gli1 inhibitor GANT61 (\#S8075) were ordered from Selleck Inc. Two mitochondrial respiratory chain inhibitors-oligomycin (\#C3007) and rotenone (\#B5462)-were ordered from ApexBio Technology. Melphalan (\#148-82-3) and bortezomib (\#179324-69-7) were ordered from MedChemExpress.

\section{Colony formation assay}

The soft agar colony formation assay was performed as previously described [12].

\section{Flow cytometry analysis}

Hoechst staining, followed by flow cytometry analysis, was performed as previously described [13]. In brief, cells were collected and re-suspended at a density of $1 \times 10^{6}$ cells $/ \mathrm{mL}$ in DMEM supplemented with $2 \%$ heatinactivated fetal bovine serum (FBS) and $10 \mathrm{mM}$ HEPES, pre-warmed to $37{ }^{\circ} \mathrm{C}$. Hoechst 33342 was added at a concentration of $5 \mu \mathrm{g} / \mathrm{mL}$. In a parallel sample aliquot, SP inhibitor verapamil (\#V4629, Sigma Aldrich LLC, US) was used at a final concentration of $5 \mu \mathrm{M}$. Cells were incubated for $2 \mathrm{~h}$ in a water bath at $37^{\circ} \mathrm{C}$ with periodic agitation. Cells were then centrifuged for $5 \mathrm{~min}$ at $400 \times 9,4^{\circ} \mathrm{C}$, and resuspended at a concentration of $1 \times 10^{7}$ cells $/ \mathrm{mL}$ in cold HBSS containing $2 \%$ FBS and $10 \mathrm{mM}$ HEPES. Propidium iodide (PI) was added to a final concentration of $5 \mu \mathrm{g} / \mathrm{mL}$ to gate-off dead cells. SP cells were examined by flow cytometry (Moflo XDP, Beckman Coulter, US). In some experiments, SP cells were sorted with the same instrument; the same was also true for MP cells' sorting.

An ALDEFLUOR assay was performed with an ALDEFLUOR kit (\#01700, STEMCELL Tech.) to examine the aldehyde dehydrogenase (ALDH) activity of cells. Cells were collected and re-suspended at a density of $1 \times 10^{6}$ cells/ $\mathrm{mL}$ in an ALDEFLUOR ${ }^{\mathrm{TM}}$ assay buffer, and $5 \mu \mathrm{L}$ of the activated
ALDEFLUOR $^{\mathrm{TM}}$ reagent was added per milliliter of the sample. In a parallel sample tube, $5 \mu \mathrm{L}$ ALDEFLUOR ${ }^{\mathrm{TM}}$ DEAB reagent was added as a control. Cells were incubated for $30 \mathrm{~min}$ at $37^{\circ} \mathrm{C}$, centrifuged for $5 \mathrm{~min}$ at $400 \times \mathrm{g}$, $4{ }^{\circ} \mathrm{C}$, and re-suspended in $0.5 \mathrm{~mL}$ of cold ALDEFLUOR ${ }^{\mathrm{TM}}$ assay buffer. Samples were analyzed by Navios flow cytometer (Beckman Coulter, USA).

For cell-cycle analysis, the cells were collected and fixed in ice-cold $70 \%$ ethanol at $4{ }^{\circ} \mathrm{C}$ overnight. Then, the cells were centrifuged and washed with PBS, and the pelleted cells were incubated with $1 \mu \mathrm{g} / \mathrm{mL}$ propidium iodide (PI) solution containing $100 \mu \mathrm{g} / \mathrm{mL}$ RNase at $37^{\circ} \mathrm{C}$ for $30 \mathrm{~min}$. The percentage of cells in each cell-cycle phase was quantified using Modfit software (Verity Software House). For the flow cytometry-based apoptosis examination, the cells were harvested, washed, and re-suspended in $100 \mu \mathrm{L}$ of binding buffer. Apoptosis cells were examined using an Annexin V-FITC/PI apoptosis kit (\#FXP018-100, 4A Biotech, China) following the manufacturer's protocol. Annexin V or PI-positive cells were considered apoptotic cells. For mitochondria analysis, cells were processed with a Mito-Traker Red kit (\#C1035, Beyotime Biotechnology, China) per the manufacturer's protocol. The samples were then analyzed by flow cytometer (Navios, Beckman Coulter, US).

\section{RNA-sequencing analysis}

Total RNA was extracted from the cells using the TRlzol reagent (Invitrogen). The RNA was qualified and quantified using an Agilent 2100 bioanalyzer (Agilent Technologies, Palo Alto, CA, USA) and a NanoDrop instrument (ThermoFisher Scientific), respectively. Nextgeneration sequencing libraries were prepared using a NEBNext ${ }^{\circledR}$ Ultra $^{T M}$ RNA library prep kit for Illumina ${ }^{\circledR}$ according to the manufacturer's protocol. RNA libraries with different indices were multiplexed and loaded on an Illumina HiSeq instrument (Illumina, San Diego, CA, USA).
A

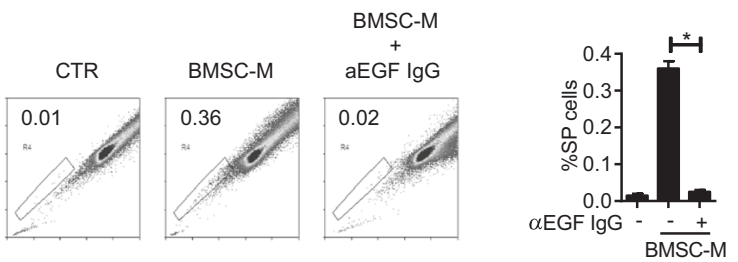

C

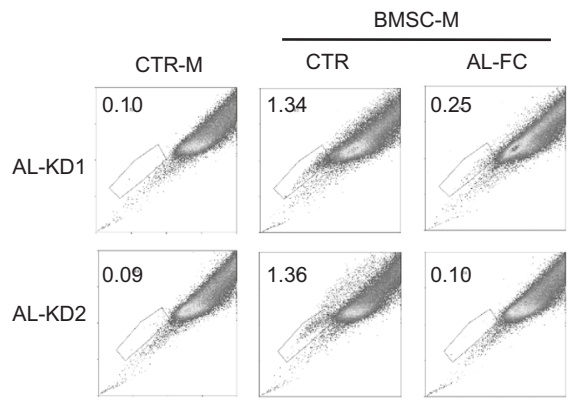

E

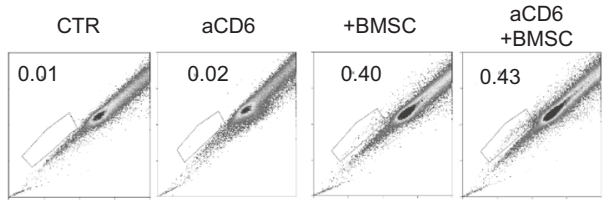

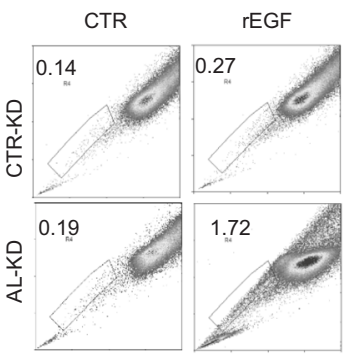

D
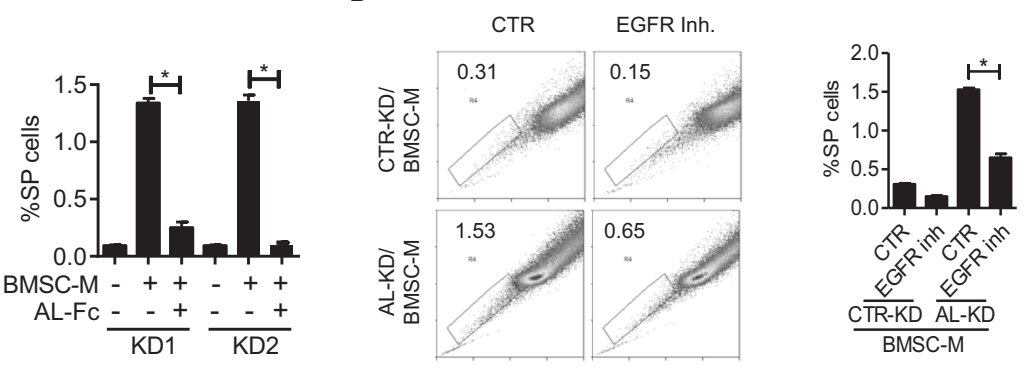
Sequencing was carried out using a $2^{\prime} 150$-bp paired-end configuration. Gene set enrichment analysis (GSEA) was conducted using GSEA software, and a heatmap was prepared using online tools (https:// software.broadinstitute.org/morpheus/). The raw data and normalized gene expression data were deposited in the Gene Expression Omnibus database under an accession number GSE182468.

\section{Reverse transcriptional quantitative PCR}

The reverse transcriptional quantitative PCR (RT-qPCR) was performed as previously described [12]. Total RNA was extracted from MM cell lines using the TRlzol reagent (Invitrogen). Reverse transcription was performed using the Evo M-MLV RT kit (Accurate Biotechnology, China). RT-qPCR was performed using the SYBR Green qPCR master mix (Bimake, China). The primer sequences are listed in Supplementary Table 1. B2M is the reference gene of mitochondria DNA.

\section{Transmission electron microscopy}

The cells were rinsed with PBS and fixed with glutaraldehyde in a cacodylate buffer. Osmication was done in $1 \%$ osmium tetroxide in a cacodylate buffer and dehydrated in an ascending series of ethanol solutions $(25,50,75,95 \%)$ for $10 \mathrm{~min}$ each, and in $100 \%$ ethanol two times for $10 \mathrm{~min}$ each. After dehydration, the cells were infiltrated with araldite: ethanol (1:1) for $2 \mathrm{~h}$, araldite:ethanol (4:1) overnight, araldite for $45 \mathrm{~min}$ at $45^{\circ} \mathrm{C}$, araldite for $45 \mathrm{~min}$ at $55^{\circ} \mathrm{C}$, and araldite to a depth of $1.5-2 \mathrm{~mm}$ before polymerization at $60^{\circ} \mathrm{C}$ overnight. Ultrathin sections $(30 \mathrm{~nm})$ of pale gold coloration cut from these blocks by an EM UC7 ultramicrotome (Leica, Inc., US) were collected onto 600-mesh copper grids, which were supported with Formvar films. The sections on the grids were examined in a HITACHI HT7700 transmission electron microscope.

\section{Animal study}

For the murine 5T-MM mouse model, murine MM 5 TGM1 cells with consistent luciferase gene expression were intravenously injected into 6-weeks-old C57BL/KawRij mice (Harlan Co. Netherland) with 2 million cells per mouse. After tumor establishment, the mice were randomly divided into 4 groups. The tumor-bearing mice were treated with PBS $(n=10)$, melphalan only $(n=9 ; 60 \mu \mathrm{g}$ per mouse every $2-3$ days, intraperitoneal injection, for a total of 4 treatments), EGFR inhibitor gefitinib only $(n=10$; $500 \mu \mathrm{g}$ per mouse every 2-3 days, intraperitoneal injection, for a total of 4 treatments), or a combination of melphalan and gefitinib $(n=9)$. Three mice from each group were sacrificed 5 days after treatment and subjected to BM SP cell examination. The remaining mice were used to monitor
A

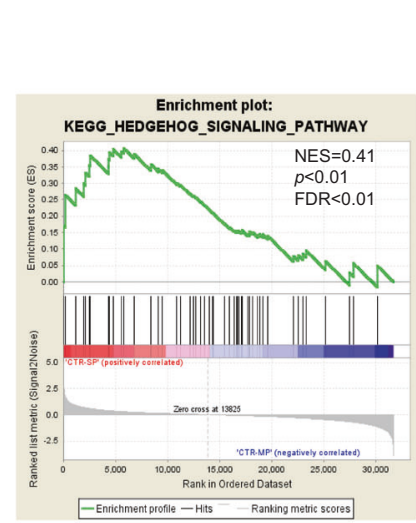

C
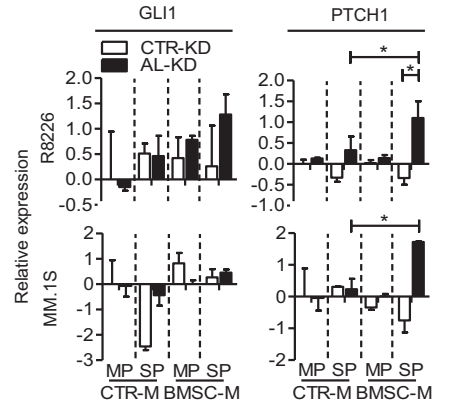

B
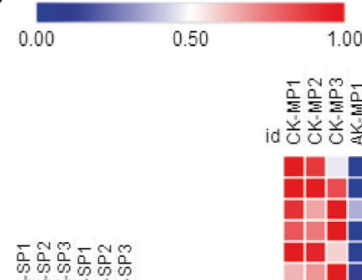

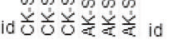

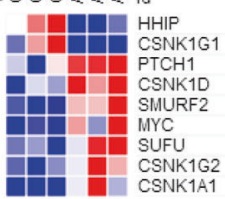

1.00

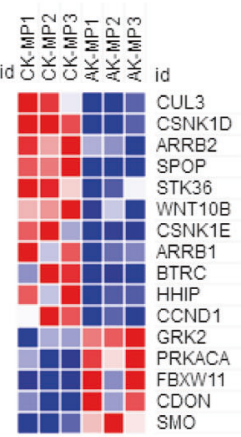

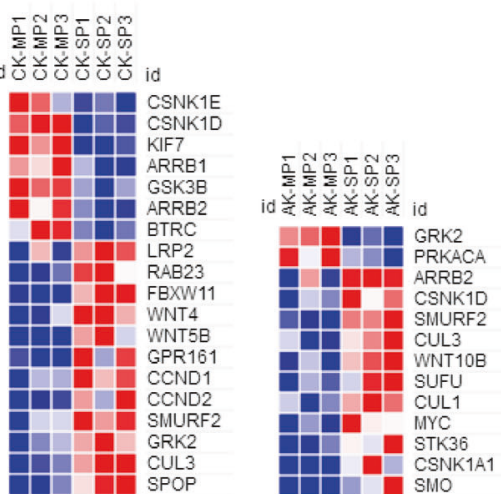

D
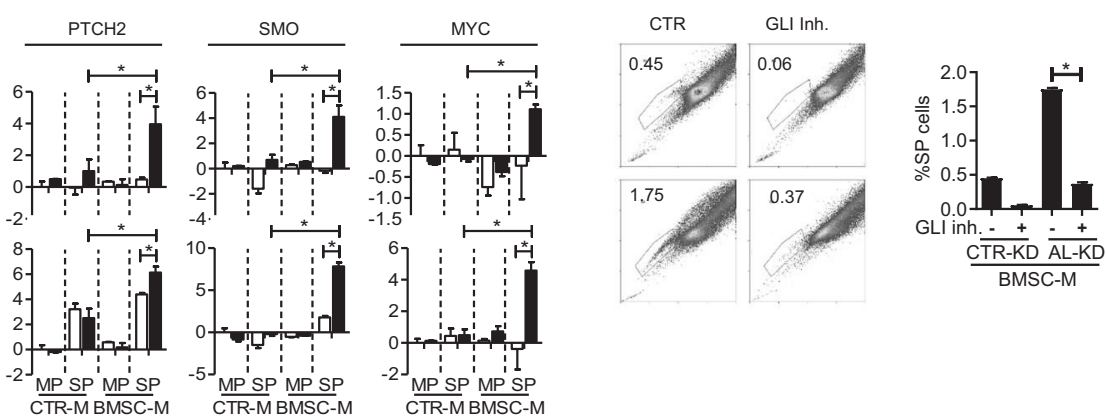

E
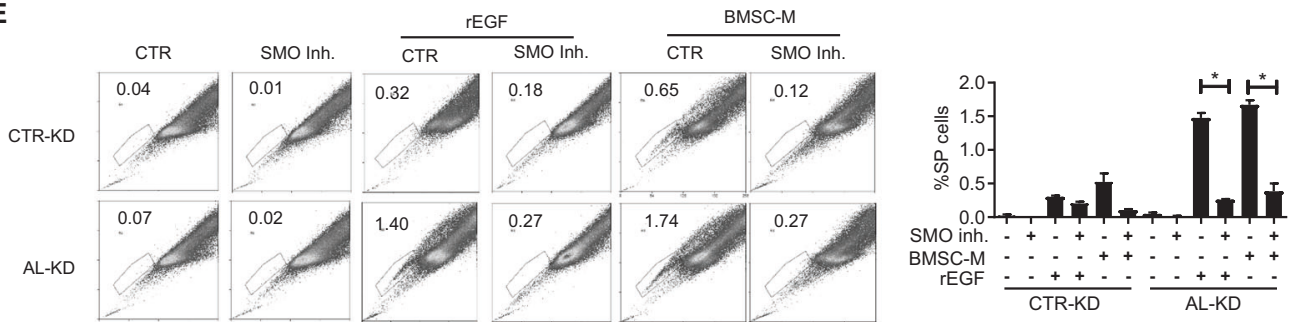

Fig. 3 ALCAM suppresses the hedgehog pathway activation. A GSEA analysis showing the hedgehog pathway gene enrichment in SP and MP cells; B Heatmap showing differentially regulated hedgehog pathway gene expression. From left to right are comparisons of CK-KD SP vs. AL-KD SP, CK-KD MP vs. AL-KD MP, CK-KD MP vs. CK-KD SP, and AL-KD MP vs. AL-KD SP. $p<0.05$ for all listed genes. C Reverse transcriptional quantitative PCR for hedgehog pathway gene expression in CTR-KD versus AL-KD MM cells. D Hoechst staining of CTR-KD or AL-KD RPMI8226 cells, cocultured with BMSC in the presence of GLI inhibitor (GNAT61, $10 \mu \mathrm{M}$ ) and the result quantification (below panel). E Hoechst staining of CTR-KD or AL-KD RPMI8226 cells, cocultured with BMSC or recombinant EGF protein (10 ng/ml) in the presence of SMO inhibitor (cyclopamine, $50 \mathrm{nM}$ ) for $24 \mathrm{~h}$ and the result quantification (right panel). Data are the mean of three independent experiments in three replicates. ${ }^{*} p<0.05$ 
treatment outcomes. Alternatively, after the 5T-MM mouse model was established, the tumor-bearing mice were divided into 3 groups and treated with PBS $(n=4)$, bortezornib $(n=6,15 \mu \mathrm{g}$ per mouse every 2-3 days, intraperitoneal injection, for a total of 4 treatments), or a combination of bortezornib and gefitinib $(n=6)$. The mice tumor burdens were examined by an in vivo luciferase assay using IVIS machinery (IVIS Spectrum In Vivo Imaging System, Perkin Elmer, US). Mouse peripheral blood was collected from the angular vein every 5 days. 5TGM1 monoclonal IgG2b protein levels in the peripheral blood were examined by ELISA kit (\#88-50430-88, ThermoFisher Scientific, US). All mouse studies complied with protocols approved by the IACUC committee of West China Hospital, Sichuan University.

\section{Statistical analysis}

Unless otherwise indicated, all data are expressed as the mean \pm standard deviation. Statistical analysis was conducted using the statistical software GraphPad Prism 8. Survival was analyzed by the Kaplan-Meier log-rank test. A $p<0.05$ was considered statistically significant.

\section{RESULTS}

ALCAM regulates myeloma side population in bone marrow microenvironment

Using shRNA lentivirus, we generated consistent ALCAMknockdown MM cells (AL-KD), as well as control knockdown
(CTR-KD). The knockdown efficacy was determined in our previous publication [12]. Hoechst staining showed that AL-KD MM cells (RPMI8226 and MM.1S) cultured in the BMSCconditioned medium (BMSC-M) had a higher ratio of SP cells than CTR-KD (Fig. 1A). AL-KD cells generated with different ALCAM shRNA sequences showed a similar result (Fig. 1B). Next, we sorted SP MM cells after Hoechst staining and labeled the cells with CellTrace Far Red fluorescence dye (Fig. 1C). Since SP MM cells alone did not survive in vitro after sorting (data not shown), we mixed labeled SP cells with MP cells and cocultured the cell mix with BMSC for 4 days. AL-KD SP cells exhibited more "diluted" fluorescence intensity than CTR-KD SP cells, a result indicating that AL-KD SP had more active cell proliferation than CTR-KD SP under coculture (Fig. 1D). We also examined the clonogenic activities of MP and SP MM cells. We used a 3D printing technology to infuse MM cells (CFSE-labeled, green) and BMSCs (CellTrace Far Red-labeled, red) into a matrix. After coculture, SP-depleted MM cells had no MM proliferation identified (Fig. 1E). Finally, in a soft agar colony formation assay, only SP MM cells cocultured with BMSC resulted in colony formation. AL-KD SP cocultured with BMSC had more colony formation than CTR-KD SP cocultured with BMSC (Fig. 1F). Such findings agreed with a previous publication that SP MM cells exhibited tumor-initiating activity [14]. Overall, our results
A

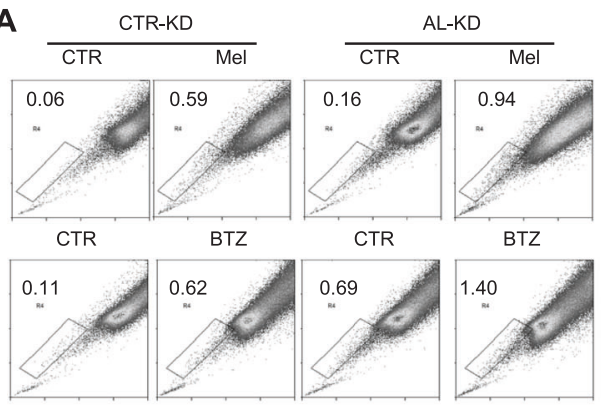

C

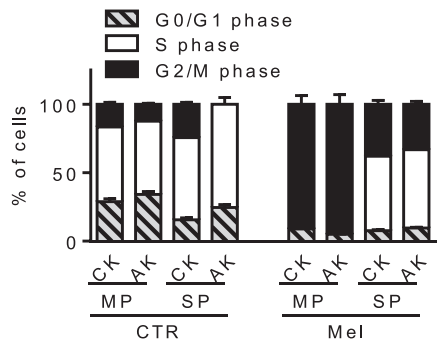

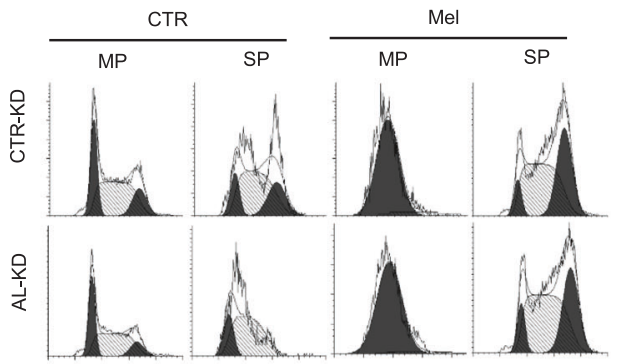

E
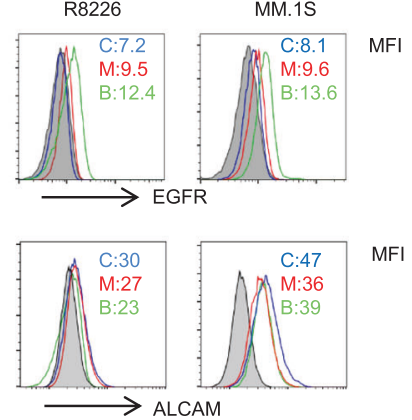

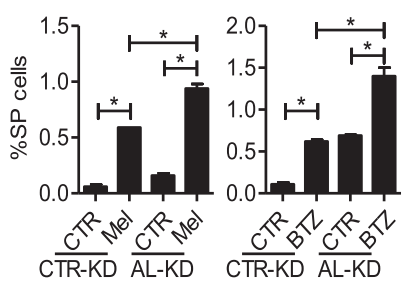

D
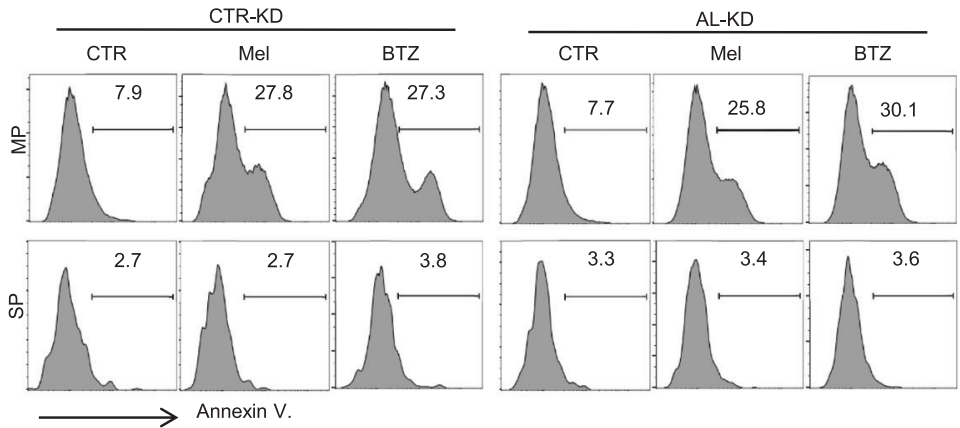

$\mathbf{F}$

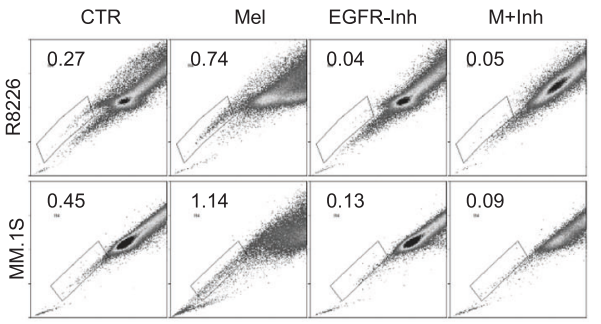

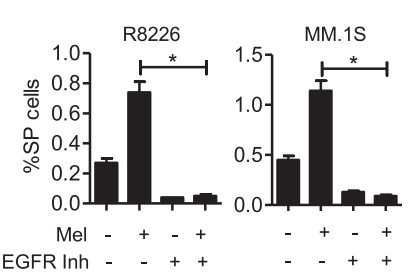

Fig. 4 ALCAM regulates myeloma chemoresistant side population in vitro. A MM cells RPMI8226, either CTR-KD or AL-KD, were treated with melphalan (Mel, $15 \mu \mathrm{M})$ or bortezomib $(\mathrm{BTZ}, 5 \mathrm{nM})$ for $24 \mathrm{~h}$. The SP cell ratio was examined by Hoechst staining. B The RPMI8226 cells were treated by melphalan as described above. The cell cycle was analyzed after Hoechst staining. C Cell-cycle quantification. D After Hoechst staining, the apoptotic cells were analyzed by annexin V staining. E ALCAM and EGFR expression on MM cells after Mel or BTZ treatment were detected by flow cytometry. MFI mean fluorescence index. F Examination of SP cells after EGFR inhibitor (gefitinib, $200 \mathrm{nM}$ ) and melphalan treatment. Data are the mean of three independent experiments in three replicates. ${ }^{*} p<0.05$. 
show that ALCAM-knockdown promotes SP MM cells in the presence of BM-derived factors. SP MM cells may contribute to tumor initiation.

\section{ALCAM-EGF/EGFR axis regulates myeloma side population}

Previously, we showed that ALCAM interacts with EGFR and inhibits EGF priming-initiated EGFR downstream cell signaling and MM clonogenicity. Thus, we hypothesized that the ALCAM-EGFR/ EGF axis regulated the abundance of SP MM cells. To test this hypothesis, we first examined the role of EGF in SP regulation. EGF blocking antibody inhibited SP population in MM cells cultured in BMSC-M (Fig. 2A), while the addition of EGF recombinant protein in the cell culture medium stimulated SP MM, particularly in AL-KD MM (Fig. 2B). Next, the addition of the ALCAM-Fc chimera fusion protein, which mimics the ALCAM function, inhibited BMSC-M stimulated SP population in AL-KD MM cells (Fig. 2C). EGFR inhibitor gefitinib inhibited the BMSC-M-stimulated SP population (Fig. 2D). Finally, we examined how the CD6 blockade affects SP MM. Previous research showed that ALCAM interacts with CD6 and mediates intercellular adhesion and migration [15]. However, the CD6 block antibody did not affect the BMSC coculturestimulated SP population (Fig. 2E). Overall, our data confirm our hypothesis that the ALCAM-EGFR/EGF axis regulates the abundance of SP MM cells. Such regulation is independent of the ALCAM-CD6 interaction.

\section{ALCAM regulate myeloma side population through the hedgehog pathway}

Previous studies showed that SP MM cells may be regulated by hematopoietic stem cell regulatory cell signaling pathways such as the Wnt/ $\beta$-catenin pathway [8], the hedgehog pathway [16], and the Notch pathway [17]. To identify ALCAM downstream cell signaling in SP MM regulation, we sorted SP and MP cells from CTR-KD and AL-KD MM in culture, and examined gene expression profiles. In general, MP and SP MM cells exhibited differentially regulated gene expressions (Supplementary Fig. 1A). Pathway enrichment analysis showed that the hedgehog pathway was altered in AL-KD MM cells, compared with CTR-KD (Fig. 3A). Differentially regulated hedgehogpathway genes were identified (Fig. 3B). We verified some hedgehog pathway gene expression in MP versus SP cells by quantitative RT-PCR. As shown in Fig. 3C, SP cells isolated from the AL-KD MM cell culture had the highest hedgehog pathway gene expression. The hedgehog pathway inhibitor inhibited the BMSC-M- or EGF recombinant protein-stimulated SP population (Fig. 3D, E). We also analyzed the Wnt/ $\beta$-catenin pathway and Notch pathway genes in MP versus SP MM cells, two pathways that have been shown to regulate $M M$ tumor-initiating cells (Supplementary Fig. 1B, C). To summarize, our data suggest that the ALCAM-EGR/EGF axis regulates SP MM cells through the hedgehog pathway.

\section{The myeloma side population is chemoresistant}

Previous research showed that SP MM cells are less sensitive than MP MM cells to chemotherapeutic agents. In our study, we found that anti-MM agents melphalan (Mel) or bortezomib (BTZ) treatment both induced an increased ratio of SP cells, particularly in AL-KD RPMI8226 MM cells (Fig. 4A). Cell-cycle analyses showed that, under the Mel treatment, SP cells still had an active cell cycle, while MP cells were arrested at the G2/M phase (Fig. 4B, C). The apoptotic assay showed that BTZ or Mel exposure could only induce apoptosis in MP cells, but not in SP cells (Fig. 4D). In our previous publication, as well as in data presented earlier in this study, we demonstrated that the ALCAM-EGFR/EGF axis regulated MM SP cells and clonogenicity. EGFR, primed with EGF, promoted MM SP cells, while ALCAM inhibited such promotion.
A
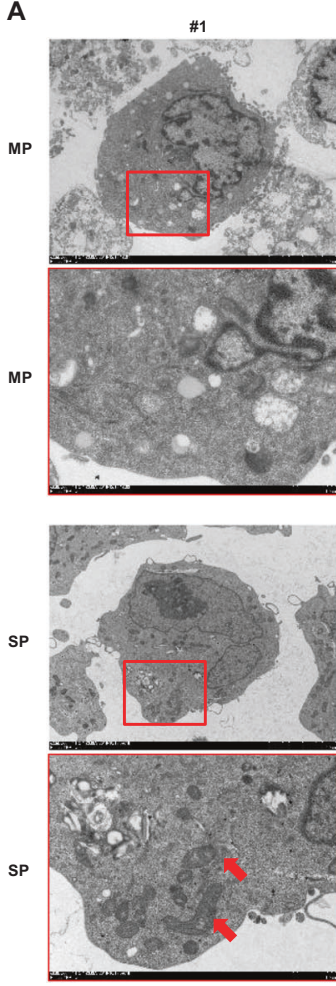
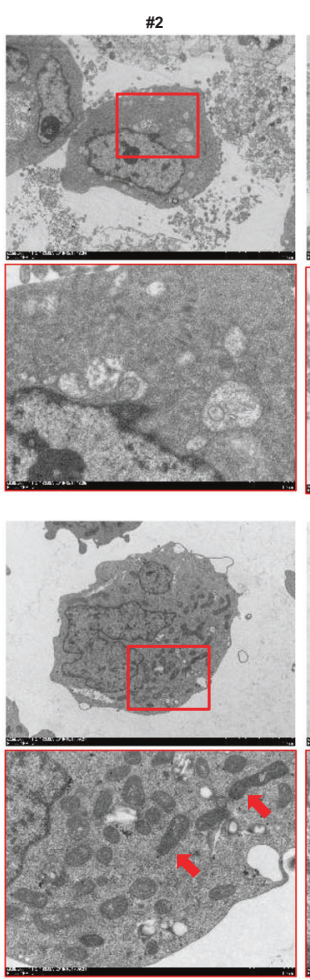

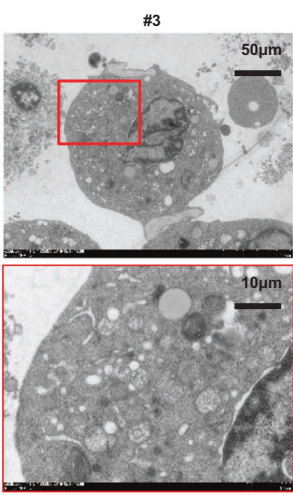

B

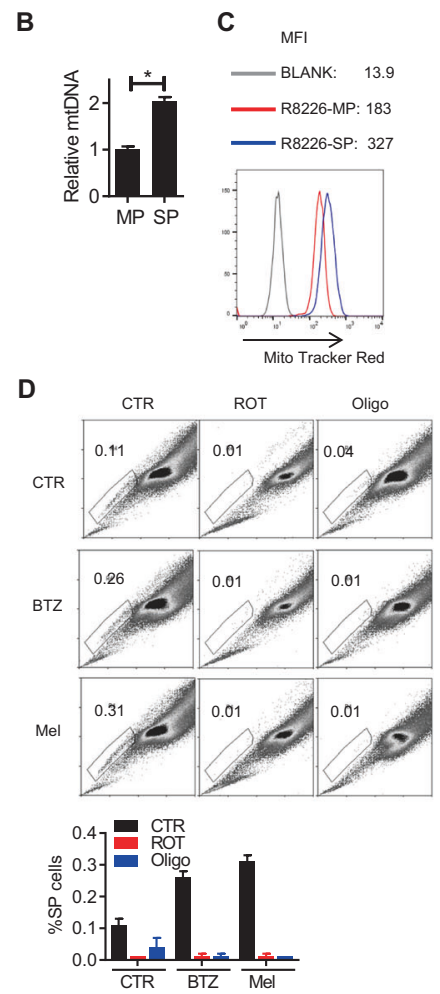

D

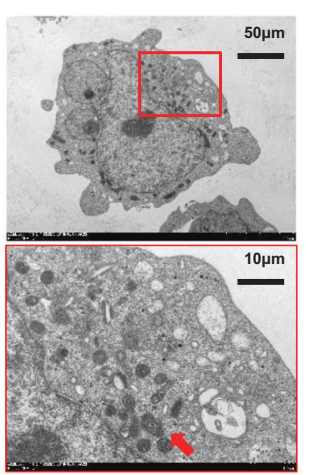

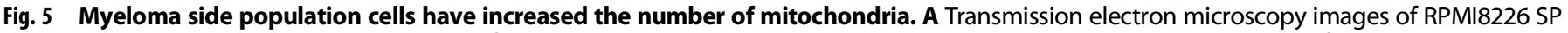

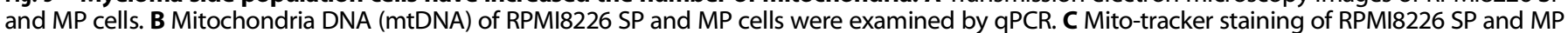

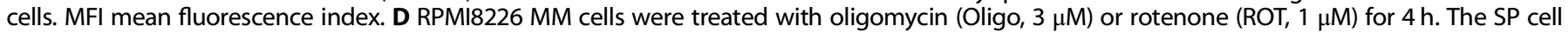
ratio was examined by Hoechst staining. Data are the mean of three independent experiments in three replicates. ${ }^{*} p<0.05$. 
A

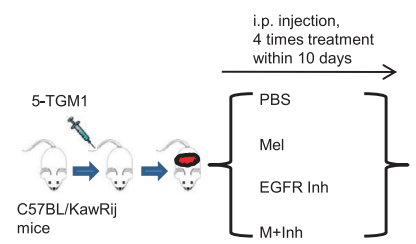

B

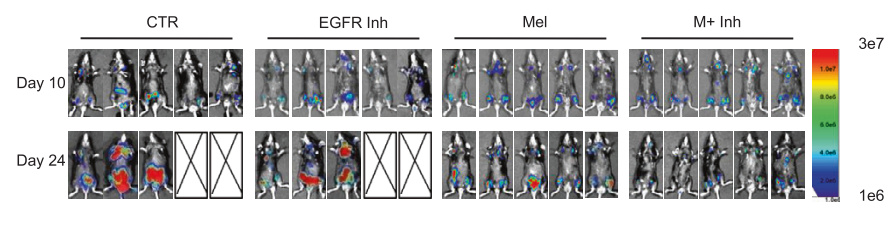

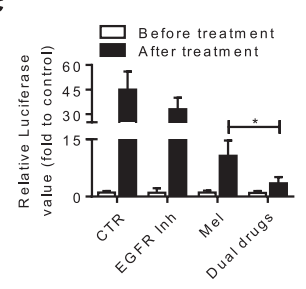

E

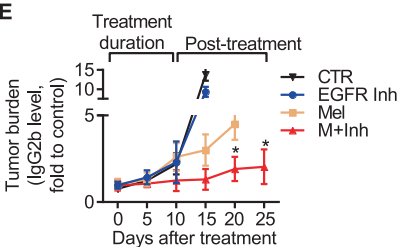

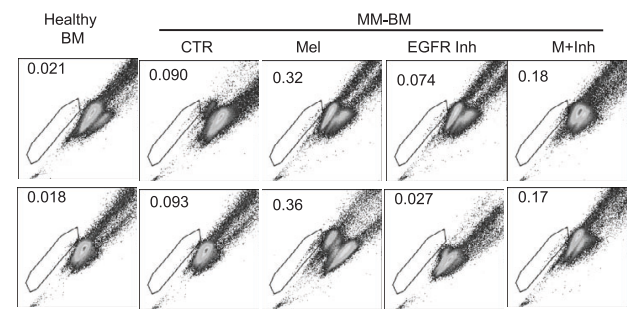

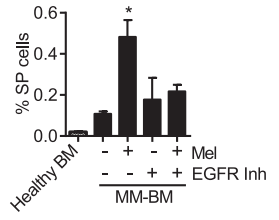

$\mathbf{F}$
G

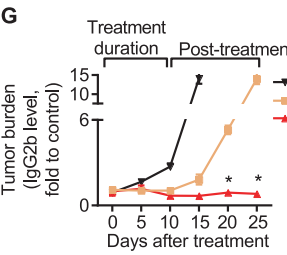

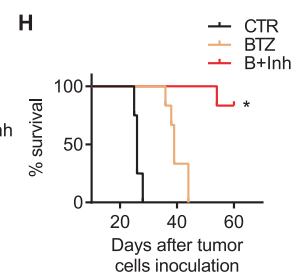

Fig. 6 EGFR-targeting therapy attenuates side population conferred myeloma chemoresistance in vivo. A Scheme graph showing the animal study to evaluate the efficacy of the combination therapy (Mel and EGFR inhibitor) in vivo. The mice were treated by intraperitoneal injection of melphalan ( $60 \mu \mathrm{g} / \mathrm{mouse}$ per time, 4 times within 10 days) or gefitinib $(500 \mu \mathrm{g} / \mathrm{mouse}$ per time, 4 times within 10 days), or a combination of both. Each group contained 8 mice. B Tumor-bearing mice were subjected to in vivo bioluminescent imaging (IVIS) before and after treatment. Five out of seven representative results are shown. C The relative luciferase activity of IVIS was calculated. D The tumorbearing mice were treated twice as described above. Then, mice BM cells were analyzed by Hoechst staining for MM SP in vivo. Two out of three representative results are shown in the left panel, and result quantification is shown in the right panel. $\mathbf{E}$ Tumor burden was evaluated by circulating lgG2b. F Treatment efficacy was evaluated by mouse survival. G MM tumor-bearing mice were treated by intraperitoneal injection of PBS, bortezomib ( $15 \mu \mathrm{g} / \mathrm{mouse}$ per time, 4 times within $10 \mathrm{days})$, or a combination of bortezomib and gefitinib (500 $\mu \mathrm{g} / \mathrm{mouse}$ per time, 4 times within 10 days). The PBS group contained 4 mice; the other treated group each contained 6 mice. Tumor burden was evaluated by circulating IgG2b. H Treatment efficacy was evaluated by mouse survival $\left({ }^{*} p<0.05\right)$.

The balance of EGFR and ALCAM expressions determined the SP ratio. Therefore, we examined ALCAM and EGFR expressions on MM cells after drug treatment. Mel or BTZ treatment-induced EGFR expression and repressed ALCAM expression (Fig. 4E). Thus, the drug-induced SP upregulation might occur due to the shift in the ALCAM and EGFR expression balance. Finally, we examined how the EGFR inhibitor gefitinib affects SP cells. We found that gefitinib inhibited melphalan-induced SP cells (Fig. 4F). We also repeated the above experiments in MM.1S cells and found similar results (Supple. Figure 2). Overall, our data show that anti-MM drug treatment induces the upregulation of chemoresistant SP MM cells. EGFR inhibition blocks chemoresistant SP MM cell generation.

\section{Myeloma side population cells have increased the number of mitochondria \\ Next, we characterized the morphological features of SP MM RPMI8226 cells. Transmission electron microscopy (TEM) showed that SP cells had significantly more mitochondria than MP cells (Fig. 5A). In addition, mitochondria DNA ( $m t D N A$ ) examination (Fig. 5B) and mito- tracker staining (Fig. 5C) both suggested that SP cells had more mitochondria than MP cells. Inhibition of the mitochondria functions strongly inhibited drug-induced SP cell upregulation (Fig. 5D). To summarize, our data show that mitochondria play a critical, but still mechanistically undetermined, role in SP MM cells.}

EGFR-targeting therapy overcomes myeloma side populationmediated drug-resistance in vivo

According to the above data, we proposed that anti-MM drug treatment would induce increased chemoresistant SP MM cells.
ALCAM-low MM had a higher capacity than ALCAM-high MM cells in generating SP cells. Therefore, ALCAM-low MM might be more resistant to anti-MM agents than ALCAM-high MM. Next, we examined SP-mediated MM chemoresistance in vivo in a 5TMM model (Fig. 6A). Tumor imaging showed that EGFR inhibition alone, which could block SP cell generation, did not affect tumor growth in vivo. Melphalan and EGFR inhibitor gefitinib combination therapy resulted in better tumor shrinkage than melphalan alone (Fig. 6B, C). Analyses of ex vivo tumor cells showed that gefitinib repressed SP MM cells (Fig. 6D). Circulating $M$ protein (Fig. 6E) and mice survival (Fig. 6F) both showed that combination therapy had a better treatment outcome compared to single-drug treatment. In particular, the combination-treated mice had a prolonged stage of stable disease after treatment. The same was true in BTZ-based treatment (Fig. 6G, H). To summarize, we showed that SP MM cells mediate drug-resistance in vivo. EGFR inhibition can therefore overcome SP-mediated MM chemoresistance.

\section{ALCAM expression in primary myeloma cells}

Finally, we examined the stemness of MM cells in primary samples. Since BM aspiration from each patient could not provide sufficient cells for Hoechst staining, we used an ALDEFLUOR assay to determine stemness cells in BM. The ALDEFLUOR assay identified cancer stem cells in vitro and in vivo based on the cell aldehyde dehydrogenase activity [18]. As shown in representative data in Fig. 7A (left) and the summary of results (Fig. $7 \mathrm{~B}$, right), MM BM had a higher ratio of $\mathrm{ALDFLUOR}^{+}$ cells than normal BM. Next, we performed an ALDEFLUOR assay 

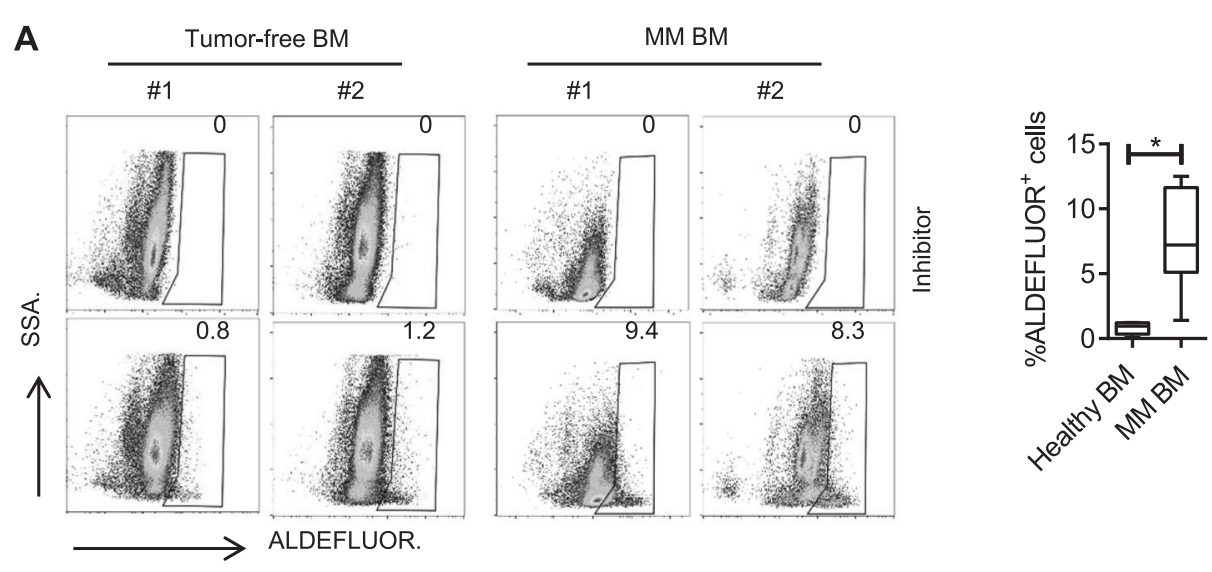

B
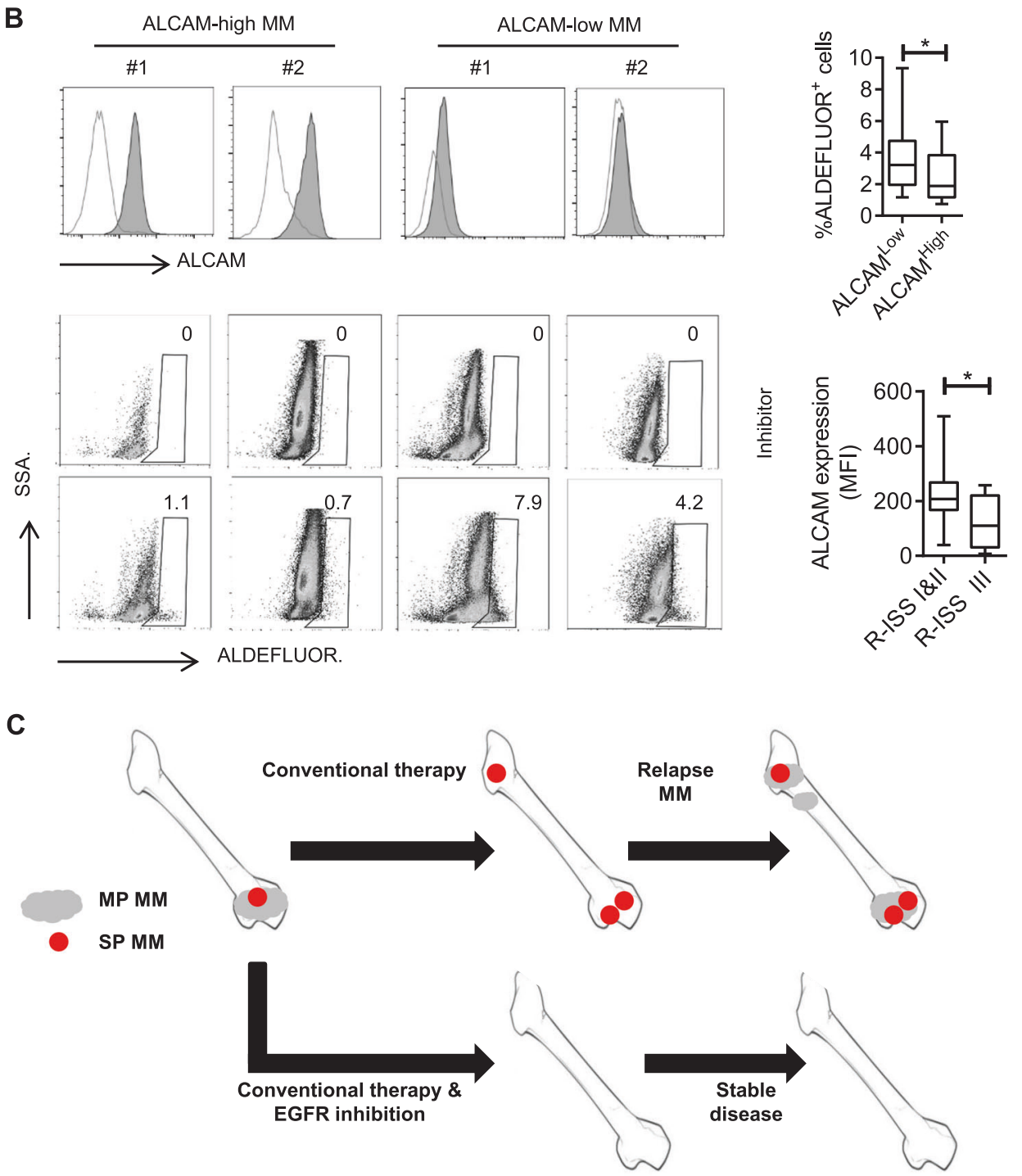

Fig. 7 ALCAM expression and stemness myeloma cells. A ALDEFLUOR analysis of BM cells from non-tumorous donors $(n=5)$ and MM patients $(n=7)$. Two representative results are shown. B BM cells from 42 newly diagnosed MM patients were analyzed by flow cytometry for ALCAM expression in CD138 ${ }^{+}$MM cells and ALDEFLUOR staining. Results show 2 representatives of ALCAM-high MM versus ALCAM-low MM, and corresponding ALDEFLUOR staining results. C Graphic model of EGFR-targeting therapy in MM treatment $\left({ }^{*} p<0.05\right)$.

in a group of primary MM cells. The patient characteristics are summarized in Supplementary Table 2. ALCAM expression in primary MM cells was determined by RT-qPCR. The patients were divided into two groups according to ALCAM expression.
The patients with ALCAM ${ }^{\text {Low }} M M$ had a higher ratio of $\mathrm{ALDFLUOR}^{+}$cells than patients with $\mathrm{ALCAM}^{\text {High }}$ MM (Fig. 7B); R-ISS III MM patients had more ALDFLUOR ${ }^{+}$cells than R-ISS I\&II patients (Fig. 7B). To summarize, our data show the existence of 
$\mathrm{ALDFLUOR}^{+}$in MM BM. The ratio of ALDEFLUOR ${ }^{+}$cells in MM BM negatively correlates with MM ALCAM expression.

\section{DISCUSSION}

Previously, we identified functional crosstalk between ALCAM and EGFR [12]. MM cells expressed both ALCAM and EGFR. ALCAM interacted with EGFR and blocked BM microenvironmentderived EGF binding to its receptor, thus inhibiting the EGFR downstream cell signaling that regulated MM clonogenicity. In this study, we further investigated the ALCAM-EGF/EGFR axis in MM drug-resistance regulation. We found that the level of ALCAM expression on MM cells correlated with the ratio of drug-resistant SP MM cells within the BM microenvironment. Anti-MM agent treatment stimulated SP MM cell generation in vitro and in vivo. ALCAM ${ }^{\text {High }} \mathrm{MM}$ cells had lower SP-genesis capacity than ALCAM ${ }^{\text {Low }}$ MM cells. The low SP-genesis capacity of ALCAM $^{\text {High }} M M$ was consistent with our previous findings that ALCAM $^{\text {High }} \mathrm{MM}$ exhibited low clonogenesis and prolonged patient survival [12].

The MM drug-resistance mechanism is complex and still under active investigation. Some researchers have introduced the concept of MM cancer stem cells (MM-CSCs) to demonstrate drug-resistance. In general, CSCs refer to a small population of malignant cells with distinguishable activities of clonogenicapacity, self-renewal, and differentiation into regular cancer cells [19]. Previous studies indicated that MM-CSCs were resistant to chemotherapy and might be a promising target to control drugresistance $[8,20]$. In many ways, MM-CSCs are still conceptual, so there has been controversy in defining and characterizing them [20]. Alternative terminologies might be used for describing those cells, such as MM stemness side population [7], MM clonogenic cells [21], or MM cancer-initiating cells [22]. Although MM-CSCs have not yet been properly defined, there are still several ways to detect them, such as via Hoechst staining [20] and an ALDEFLUOR assay [18], both of which were used in our study.

It is generally believed that CSCs may have important therapeutic implications [23]. Our results suggest that a combination therapy with an EGFR inhibitor and anti-MM agents might be promising for MM treatment. EGFR inhibitor alone has no anti-MM activity in vitro and in vivo. A previous cell line study suggested that EGFR inhibitor gefitinib had no anti-MM activity against MM cells with KRAS, NRAS, or BRAF mutations [24]. An earlier phase II study of VEGFR/EGFR inhibitor Zactima in MM also showed that the inhibitor was well tolerated in patients but could not reduce the patients' M protein [25]. According to our findings, EGFR inhibition repressed drug-resistant SP MM cells, which only accounted for a small number of total neoplasmic cells. Therefore, EGFR inhibitor should be used in combination with other anti-MM agents. Our findings also suggest that, during the combination therapy, anti-MM agents were used to eradicate most MM cells, while EGFR inhibition targeted a small number of drug-resistant MM cells. Combination therapy might increase the duration of complete remission after treatment and decrease the frequency of treatment. To the best of our knowledge, there are no ongoing clinical trials testing EGFR inhibition and anti-MM combination therapy. This lack of research is probably because EGFR expression is low and EGFR gene mutation is rare in MM. Our findings provide evidence to support such clinical studies. Further, we also believe that MRD status after treatment and CR duration time are the two critical factors needed to evaluate the combination therapy.

\section{DATA AVAILABILITY}

The data sets generated and/or analyzed during the current study are available from the corresponding author on reasonable request.

\section{REFERENCES}

1. Raab MS, Podar K, Breitkreutz I, Richardson PG, Anderson KC. Multiple myeloma. Lancet. 2009;374:324-39.

2. Lonial S, Anderson KC. Association of response endpoints with survival outcomes in multiple myeloma. Leukemia. 2014;28:258-68.

3. Mitsiades CS, Davies FE, Laubach JP, Joshua D, San Miguel J, Anderson KC, et al. Future directions of next-generation novel therapies, combination approaches, and the development of personalized medicine in myeloma. J Clin Oncol. 2011;29:1916-23.

4. Matsui W, Huff CA, Wang Q, Malehorn MT, Barber J, Tanhehco Y, et al. Characterization of clonogenic multiple myeloma cells. Blood. 2004;103:2332-6.

5. Franqui-Machin R, Wendlandt EB, Janz S, Zhan F, Tricot G. Cancer stem cells are the cause of drug resistance in multiple myeloma: fact or fiction? Oncotarget. 2015;6:40496-506.

6. Guo W, Wang $H$, Chen $P$, Shen X, Zhang B, Liu J, et al. Identification and characterization of multiple myeloma stem cell-like cells. Cancers. 2021;13:3523

7. Du J, Liu S, He J, Liu X, Qu Y, Yan W, et al. MicroRNA-451 regulates stemness of side population cells via PI3K/Akt/mTOR signaling pathway in multiple myeloma. Oncotarget. 2015;6:14993-5007.

8. Yang Y, Shi J, Gu Z, Salama ME, Das S, Wendlandt E, et al. Bruton tyrosine kinase is a therapeutic target in stem-like cells from multiple myeloma. Cancer Res. 2015;75:594-604.

9. Fan JL, Zhang J, Dong LW, Fu WJ, Du J, Shi HG, et al. URI regulates tumorigenicity and chemotherapeutic resistance of multiple myeloma by modulating IL- 6 transcription. Cell Death Dis. 2014;5:e1126.

10. Gao M, Bai H, Jethava $Y, W u ~ Y$, Zhu Y, Yang $Y$, et al. Identification and characterization of tumor-initiating cells in multiple myeloma. J Natl Cancer Inst. 2020;112:507-15.

11. Lehmann JM, Riethmuller G, Johnson JP. MUC18, a marker of tumor progression in human melanoma, shows sequence similarity to the neural cell adhesion molecules of the immunoglobulin superfamily. Proc Natl Acad Sci USA. 1989;86:9891-5.

12. Luo $H$, Zhang $D$, Wang $F$, Wang $Q$, Wu $Y$, Gou $M$, et al. ALCAM-EGFR interaction regulates myelomagenesis. Blood Adv. 2021;5:5269-82.

13. Goodell MA, Brose K, Paradis G, Conner AS, Mulligan RC. Isolation and functional properties of murine hematopoietic stem cells that are replicating in vivo. J Exp Med. 1996;183:1797-806.

14. Ai L, Mu S, Sun C, Fan F, Yan H, Qin Y, et al. Myeloid-derived suppressor cells endow stem-like qualities to multiple myeloma cells by inducing piRNA-823 expression and DNMT3B activation. Mol Cancer. 2019;18:88.

15. von Lersner A, Droesen L, Zijlstra A. Modulation of cell adhesion and migration through regulation of the immunoglobulin superfamily member ALCAM/CD166. Clin Exp Metastasis. 2019;36:87-95.

16. Peacock CD, Wang Q, Gesell GS, Corcoran-Schwartz IM, Jones E, Kim J, et al. Hedgehog signaling maintains a tumor stem cell compartment in multiple myeloma. Proc Natl Acad Sci USA. 2007;104:4048-53.

17. Jundt F, Probsting KS, Anagnostopoulos I, Muehlinghaus G, Chatterjee M, Mathas $\mathrm{S}$, et al. Jagged1-induced Notch signaling drives proliferation of multiple myeloma cells. Blood. 2004;103:3511-5.

18. Zhou W, Yang $Y, G u Z$, Wang $H$, Xia J, Wu X, et al. ALDH1 activity identifies tumorinitiating cells and links to chromosomal instability signatures in multiple myeloma. Leukemia. 2014;28:1155-8.

19. Reya T, Morrison SJ, Clarke MF, Weissman IL. Stem cells, cancer, and cancer stem cells. Nature. 2001;414:105-11.

20. Abe M, Harada T, Matsumoto T. Concise review: defining and targeting myeloma stem cell-like cells. Stem Cells. 2014;32:1067-73.

21. Wen J, Tao W, Kuiatse I, Lin P, Feng Y, Jones RJ, et al. Dynamic balance of multiple myeloma clonogenic side population cell percentages controlled by environmental conditions. Int J Cancer. 2015;136:991-1002.

22. Su J, Zhang L, Zhang W, Choi DS, Wen J, Jiang B, et al. Targeting the biophysical properties of the myeloma initiating cell niches: a pharmaceutical synergism analysis using multi-scale agent-based modeling. PLoS ONE. 2014;9:e85059.

23. Batlle $E$, Clevers $H$. Cancer stem cells revisited. Nat Med. 2017:23:1124-34.

24. Chen Y, Huang R, Ding J, Ji D, Song B, Yuan L, et al. Multiple myeloma acquires resistance to EGFR inhibitor via induction of pentose phosphate pathway. Sci Rep. 2015;5:9925.

25. Kovacs MJ, Reece DE, Marcellus D, Meyer RM, Mathews S, Dong RP, et al. A phase II study of ZD6474 (Zactima, a selective inhibitor of VEGFR and EGFR tyrosine kinase in patients with relapsed multiple myeloma-NCIC CTG IND.145. Invest New Drugs. 2006;24:529-35.

\section{ACKNOWLEDGEMENTS}

This work was supported by grants to $Y Z$ from the National Natural Science Foundation of China (Nos. 81870157, 82070219, and 81670188), and the Sichuan 
10

University Faculty Start Fund. This work was also supported by grants to TN from Incubation Program for Clinical Trials (No. 19HXFH030), Achievement Transformation Project (No. CGZH21001), 1-3-5 Project for Disciplines of Excellence, West China Hospital, Sichuan University (No. ZYJC21007), and Translational Research Grant of NCRCH (No. 2021WWB03)

\section{AUTHOR CONTRIBUTIONS}

$Y Z$ initiated and supervised the research, and prepared the manuscript. TN supervised the research. $\mathrm{FW}, \mathrm{DZ}$, and $\mathrm{HL}$ performed the majority of the experiments, data analyses, and prepared the manuscript. JH, HD, JX, ZL, YG, XZ, $Y Q, L Z, Y D, F C, T L$, and TN assisted with the experiment work and provided crucial suggestions. All authors have reviewed and approved the final version of this manuscript.

\section{COMPETING INTERESTS}

The authors declare no competing interests.

\section{ADDITIONAL INFORMATION}

Supplementary information The online version contains supplementary material available at https://doi.org/10.1038/s41419-022-04556-8.
Correspondence and requests for materials should be addressed to Ting Niu or Yuhuan Zheng.

Reprints and permission information is available at http://www.nature.com/ reprints

Publisher's note Springer Nature remains neutral with regard to jurisdictional claims in published maps and institutional affiliations.

\section{cc) (i)}

Open Access This article is licensed under a Creative Commons Attribution 4.0 International License, which permits use, sharing, adaptation, distribution and reproduction in any medium or format, as long as you give appropriate credit to the original author(s) and the source, provide a link to the Creative Commons license, and indicate if changes were made. The images or other third party material in this article are included in the article's Creative Commons license, unless indicated otherwise in a credit line to the material. If material is not included in the article's Creative Commons license and your intended use is not permitted by statutory regulation or exceeds the permitted use, you will need to obtain permission directly from the copyright holder. To view a copy of this license, visit http://creativecommons. org/licenses/by/4.0/.

(c) The Author(s) 2022 